\title{
OPEN \\ Control of decoherence with no control
}

\section{SUBJECT AREAS:}

ATOMIC AND

MOLECULAR PHYSICS

QUANTUM PHYSICS

OPTICS AND PHOTONICS

STATISTICAL PHYSICS, THERMODYNAMICS AND NONLINEAR DYNAMICS

Received

11 June 2013

Accepted

4 September 2013

Published

25 September 2013

Correspondence and requests for materials should be addressed to L.-A.W. (lianao_wu@ ehu.es)
Jun Jing ${ }^{1,2}$ \& Lian-Ao $\mathrm{Wu}^{2,3}$

\begin{abstract}
${ }^{1}$ Institute of Theoretical Physics and Department of Physics, Shanghai University, Shanghai 200444, China, ${ }^{2}$ Department of Theoretical Physics and History of Science, The Basque Country University (EHU/UPV), PO Box 644, 48080 Bilbao, ${ }^{3}$ Ikerbasque, Basque Foundation for Science, 48011 Bilbao Spain.
\end{abstract}

A common philosophy in control theory is the control of disorder by order. Control of decoherence is no exception; strategies aimed at suppressing quantum decoherence adopt this point of view. Here we predict an anomalous phenomenon in open quantum systems-control of disorder by (even more) disorder. It is shown that suppression of decoherence can be achieved using the most disordered white noise field, specifically a white Poissonian noise field. This phenomenon seems to be another anomaly in quantum mechanics and may offer a new strategy in quantum control practices.

D

ecoherence is the deterioration of quantum information in a system due to inevitable interactions with the environment or bath ${ }^{1-5}$. Suppression of decoherence is one of the paramount challenges in quantum control practices and requires accurate control of the system dynamics. Here we predict an anomaly: suppression of decoherence can be be achieved using uncontrollable white noise fields. By increasing the strength of noise signals, a two-level system becomes less coupled to its environment and even remains in the initial state for a period of time. The aberrant effect reveals a new physical mechanism in quantum control theory, and in practice may offer the possibility of control by uncontrollable white noise. We term the phenomenon as control of decoherence with no control.

Noise is a source of disorder. White noise, whose spectrum has equal power within any equal interval of frequencies, is the extreme of disorder (in comparison with coloured noise). Over a decade ago, people began to notice in classical systems that noise leads not only to nuisance but also to advantages. A remarkable example is that an external coloured noise can suppress the intrinsic white noise ${ }^{6,7}$. While it is a surprise, the phenomenon fits well with the common philosophy - control of disorder by order. This philosophy has been carried out in classical noise control and extended to suppression of decoherence in quantum dynamical processes. External field control of quantum decoherence dates back to the spin echo technique ${ }^{8}$. This technique is used to suppress the inhomogeneous spin dephasing by applying a $\pi$ inversion pulse and has been developed to tackle general decoherence ${ }^{9-17}$. However, the philosophy, control of disorder by order, remains the same for both the classical and the quantum mechanical processes. Now the predicted anomaly is opposite to the common philosophy; the most disordered white noise is used to control less disordered decoherence, which is characterized by a quantum stochastic process with coloured noise or by a non-zero correlation function over finite time. Seeing that the setting in use is exclusively quantum mechanical, it appears that the phenomenon is another anomaly in quantum systems.

Quantum mechanically, the dynamical process of a system plus its environment is governed by the total Hamiltonian,

$$
H_{\mathrm{tot}}=H_{\mathrm{S}}(t)+H_{\mathrm{B}}+H_{\mathrm{SB}},
$$

where $H_{\mathrm{S}}(t)$ and $H_{\mathrm{B}}$ are the system Hamiltonian, embedded with white noise, and the environment Hamiltonian, respectively. The system dynamics is normally characterized by master equations. The system-bath interaction $H_{\mathrm{SB}}$ is the source of decoherence.

\section{Results}

Protocol of decoherence suppressions with no control. We now introduce our protocol of decoherence suppressions, in particular suppression of dissipation. Dissipation is a decoherence process caused by the exchange of energy between a system and the environment. Provided that a two-level system is in its excitation state, the environment induces the system to give off energy and to decohere to the ground state [Fig. 1(a)]. If nature happens to have the white noise which we have required above, the decoherence can be suppressed spontaneously [Fig. 1(b)]. This required noise is described by white noise $c(t)=\eta(J, W, t)$, in particular the biased Poissonian white noise with the strength $J^{18}$. We name the average time interval between two neighbour noise signals as $1 / W \equiv T / n$, where $T$ is a time scale, different for variant systems, and $n$ is the noise arrival 


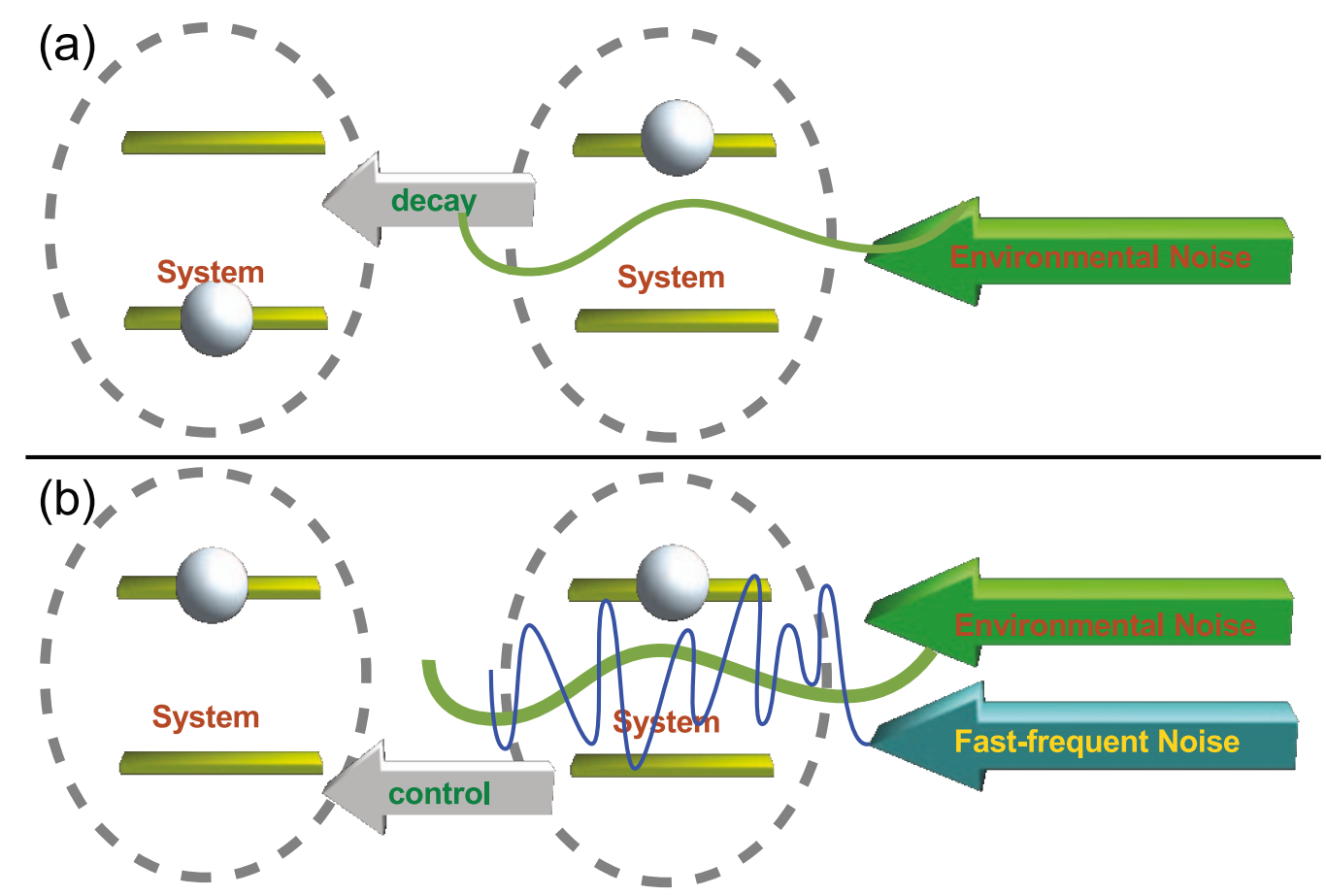

Figure 1 Sketches of (a) qauntum dissipation of a two-level system induced by its environment, (b) dissipation suppressed by white noise.

number ${ }^{18}$. When $1 / W$ goes to zero, $c(t)$ corresponds to the continuous-time white noise process, where $J$ is the only parameter. If $1 / W$ is finite, $c(t)$ can be the biased Poissonian white shot noise, which is essentially different from well-controlled, idealized or non-idealized, pulse sequences. We can tune the parameter $W$ towards the continuous time limit. In what follows, we will study the system responses to the noise $c(t)$.

Fidelity preserved by white noise. Consider a dissipative model for the two-level system, described by a non-Hermitian Hamiltonian in the exact quantum Stochastic Schrödinger equation [See Method] ${ }^{19,20}$,

$$
H_{\mathrm{ss}}(t)=[\omega+c(t)] \sigma_{z} / 2+i z_{t}^{*} g \sigma_{-}-i g Q \sigma_{+} \sigma_{-},
$$

where $\omega$ is the bare-energy spacing and $g$ is the coupling strength between system and environment. $c(t)$ is the above-mentioned white noise signal and $Q$ satisfies a nonlinear differential equation $\dot{Q}(t)=g \gamma / 2+[-\gamma+i \omega+i c(t)] Q+g Q^{2}$, with a boundary condition $Q(0)=0{ }^{17}$. The correction function of this process is $G(t, s)=$ $\frac{\gamma}{2} e^{-\gamma|t-s|}$. Here $\gamma$ characterizes the environmental memory in the Ornstein-Uhlenbek process and is inversely proportional to the environmental memory time. The values of $\gamma$ can be used to somehow determine the degree of non-Markovianity. The larger $\gamma$ is, the more Markovian the environment is. $\gamma \rightarrow \infty$ corresponds to the white noise model and indicates the Markov limit. This colored noise is formulated as $z_{t+\Delta t}^{*}=z_{t}^{*}-\gamma z_{t}^{*} \Delta t+\sqrt{\Delta t / 2} \gamma w^{*}$, where $w^{*}$ is a complex Wiener process ${ }^{17}$.

Suppose that the system initial state is $\left|\psi_{0}\right\rangle=|1\rangle$. The fidelity $\mathcal{F}(t) \equiv \sqrt{\left\langle\psi_{0}\left|\rho_{t}\right| \psi_{0}\right\rangle}$, qualifying the survival probability, evolves according to

$$
\mathcal{F}(t)=e^{-\int_{0}^{t} d s \mathcal{R}[Q(s)]},
$$

where $\mathcal{R}[\cdot]$ is the real part of the input function ${ }^{17}$. Below we will numerically study the noise effects on fidelity during time courses.

Figures 2 show $\mathcal{F}(t)$ vs time for $\gamma=0.2$ and 0.5 , subject to different $c(t)$. Suppression of dissipation is excellent in all cases with bigger $W$, in particular for less Markovian environments. Different values of $W$ represent different physics. Smaller $W$ corresponds to a sequence of noise shots with random amplitudes and sparser random arrival moments, as illustrated by $W=200 / T$. It suppresses dissipation to some extent, but not as efficient as bigger $W$. When $W$ is big enough, all different values of $W$ tend to result in the same fidelity and give an identical curve, as exemplified by the red solid curves with $W=$ $1000 / T$. These curves correspond to the continuous-time white noise.

The quality of suppression depends mostly on the strength $J$ of the continuous-time white noise and environmental non-Markovianity $\gamma$. The two figures show clearly that the larger $J$ is, the better the quality of suppression is. The parameter $\gamma$ seriously influences the quality of suppression as well. Suppression becomes worse at $\gamma=0.5$ than at $\gamma=0.2$, and even worse when the environment is more Markovian. Eventually, suppression is invalid in a complete Markovian environment $(\gamma \rightarrow \infty)$, corresponding to quantum white noise. Significantly, it shows that white noise cannot suppress white noise, i.e. complete disorder cannot suppress complete disorder.

Now we look into the detailed roles that the parameters $J$ and $W$ play. Figure 3 plots the fidelity contour as a function of $J$ and $W$ at two time moments $t=50 \mathrm{~T}$ and $t=100 \mathrm{~T}$. The regions where $\mathcal{F}>0.99$ are highlighted. The fidelity is saturated at $W>600 / T$ for both figures, where a discrete random pulse sequence becomes the continuous-time white noise. While the fidelity is excellent for bigger values of $J$, it seems not to be saturated with $J$. The bigger $J$ is, the better the fidelity is.

The numerical results presented by figures could be valid for various physical systems with corresponding characteristic values of $T$. For example, $\omega$ is approximately $10^{9}-10^{10} \mathrm{~Hz}$ in a superconducting flux qubit ${ }^{21}$. The relaxation time is $T_{1} \approx 1 \mu$ s such that the time scale $T \approx 5 \mathrm{~ns}$ and the dimensionless $\omega T \approx 5$ as taken in Fig. 2 . The required noise strength $J$ should be more than $10^{9} \mathrm{~Hz}$ in order to successfully suppress decoherence.

\section{Discussion}

The perfect suppression could be justified by the following argument. By integrating over equation (5) (see Methods), for a long time limit, one can always write, 
(a) $\gamma=0.2$

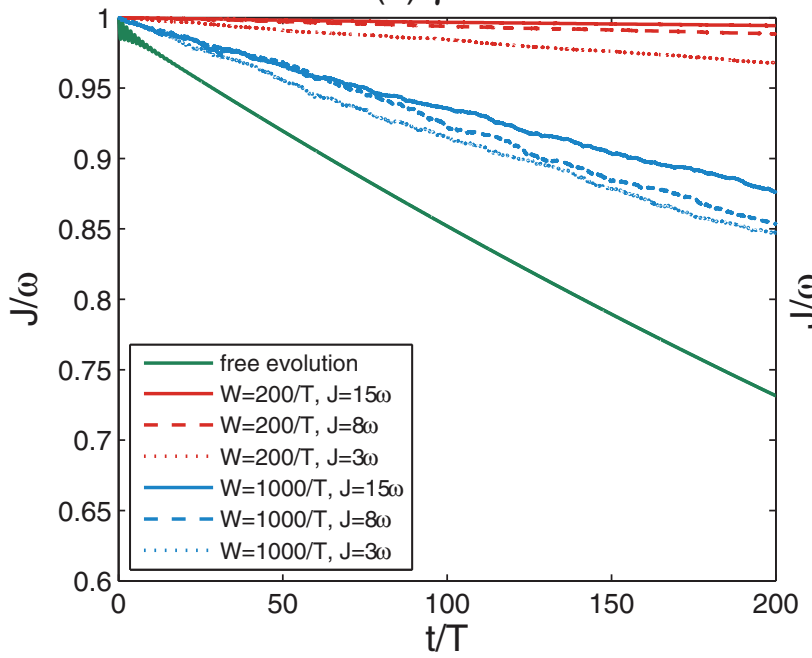

(b) $\gamma=0.5$

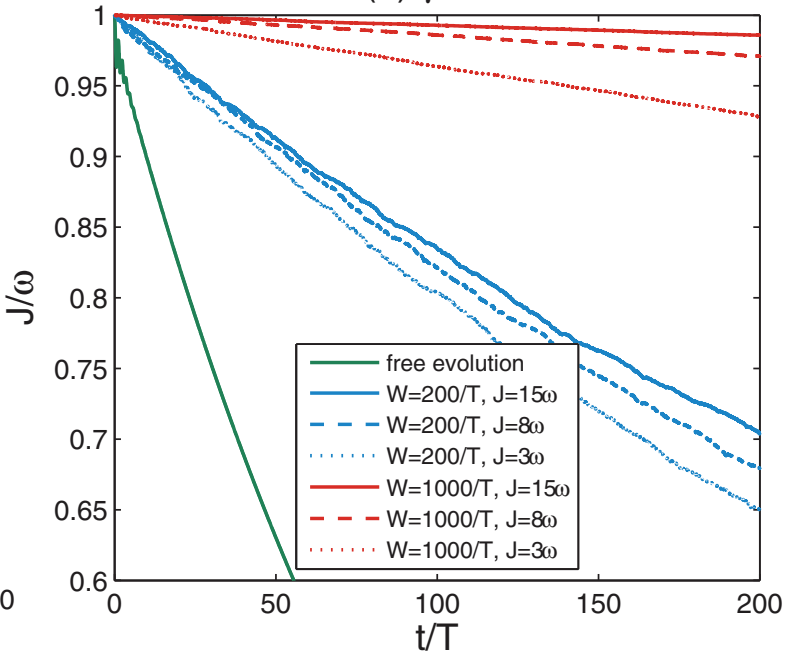

Figure $2 \mid \mathcal{F}(\boldsymbol{t})$ vs time. The environmental memory parameter $\gamma$ is taken as 0.2 and 0.5 respectively. We compare the free dynamics (green-solid curves) with those subject to different noises, red curves to $W=1000 / T$ and blue to $W=200 / T$. The solid, dashed and dotted curves represent different strengths $J=15 \omega, 8 \omega$ and $J=3 \omega$ respectively. Here $\omega T=5$ and $g=0.4 \omega$.

$$
\left\langle\psi_{0} \mid \psi_{\infty}\right\rangle-1=\sum_{\alpha \beta} \int_{0}^{\infty} N_{\alpha \beta}(t) h_{\alpha \beta}(t) d t,
$$

where $\alpha$ 's denote a set of complete bases. Here $N(t)$ and $h(t)$ represent a noisy matrix and a system dynamical matrix (see example in Methods). If each nonzero element of $N$ is a fast-varying noise function of time and the corresponding element in $h$ is much slower and weaker, the integral of each term $N_{\alpha \beta}(t) h_{\alpha \beta}(t)$ could be zero. In our model, $N(t) \propto e^{-i \int_{0}^{t} c(s) d s}$ and $h(t)=-Q(t)\left\langle\psi_{0} \mid \psi_{t}\right\rangle$ are c-numbers. For bigger values of $J, h(t)$ is slower and weaker than $N(t)$. When $h(t)$ is such slow in comparison with $N(t)$ that it can be treated as time independent, it is easily to prove $\int_{0}^{\infty} N(t) h(t) d t=0$ by using the properties of a continuous-time white noise.

The signal $c(t)$ mimics natural white noise and is also associated with dephasing processes. Our results demonstrate that the existence of noise $c(t)$ (or dephasing) significantly inhibits the dissipation. This well explains the reality where the relaxation time $T_{1}$ is longer than the dephasing time $T_{2}$ for all systems; dephasing has suppressed dissipation spontaneously.

It is easy to discriminate our approach from the well-discussed dynamical decoupling, e.g. ${ }^{1-13}$, since the latter control method is realized through designed sequences of pulses in frequency or the arrival time whereas white noise is ubiquitous and beyond artificial control. Yet it is also dramatically different from the stochastic resonance $^{22-24}$, where a dynamical system is subject to both periodic forcing (for classical systems) or Floquet Hamiltonian (for quantum systems) and random noise may show a resonance or coherent behavior which is absent when either the periodical driving or the perturbation is absent. The stochastic resonance is achieved when the noise amplitude is optimized so that it is not too weak to attain the threshold of the system signal detector and is not too strong to (a) $t=50 T$

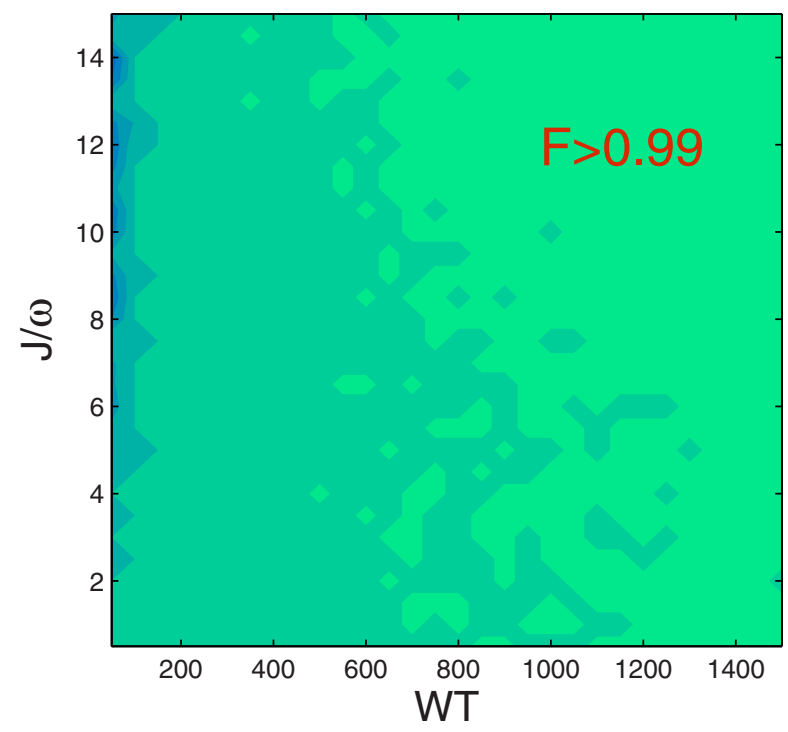

(b) $t=100 T$

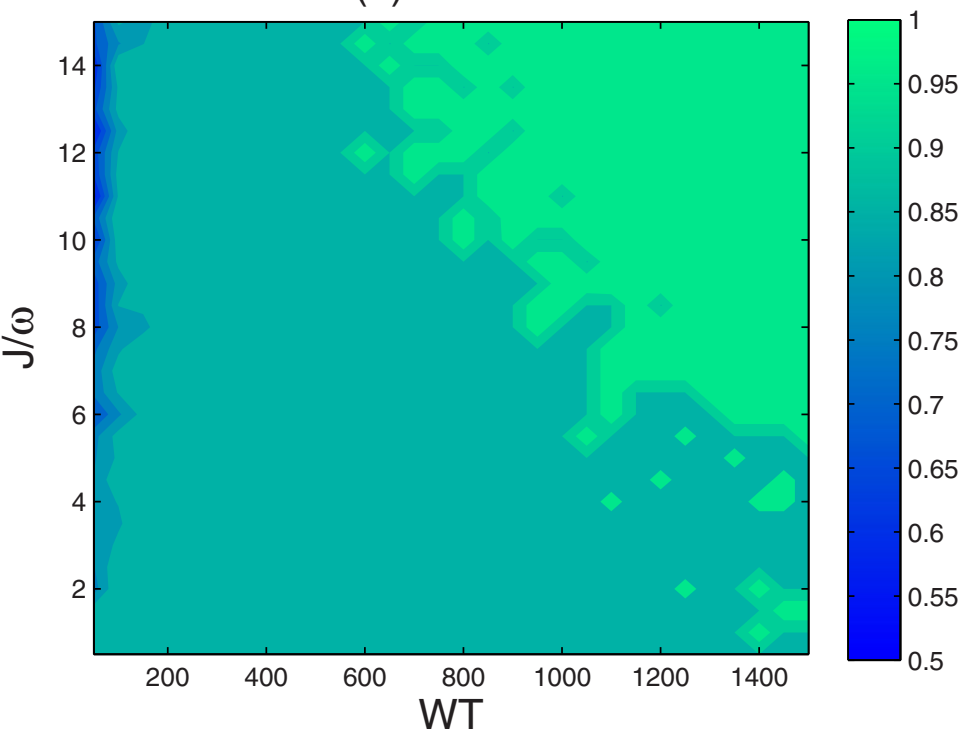

Figure $3 \mid$ Parameter thresholds and effective regions at two time moments. Here $\omega T=5$ and $g=0.4 \omega$. Fidelity where $\mathcal{F}>0.99$ is distinguished. The non-Markovian environment is characterized by $\gamma=0.2$. 
overwhelm the system coherent behavior. On the contrary, our control works when the amplitude of the white noise $J$ is above a lower bound. The stronger noise is, the better the control is. More precisely, all known control approaches fit well with the philosophy-control of disorder by order, while ours, control of disorder by even more disorder, is opposite.

\section{Methods}

Consider the system-bath interaction $H_{\mathrm{SB}}=L B^{\dagger}+L^{\dagger} B$ for simplicity. The exact stochastic Schrödinger equation is ${ }^{19,20,25}$ :

$$
i \partial_{t} \psi_{t}\left(z^{*}\right)=H_{\mathrm{ss}}(t) \psi_{t}\left(z^{*}\right) .
$$

where $H_{\mathrm{ss}}=H_{S}(t)+i L z_{t}^{*}-i L^{\dagger} \bar{O}$ is an exact system Hamiltonian and $L=g \sigma_{-}$for our two-level system. $\bar{O}$ is a combination of system operators and environmental noises satisfying consistency conditions ${ }^{19}$. Each quantum trajectory is accompanied by a special process $z^{*}$, and the system density matrix is given by $\rho_{t}=M\left[\left|\psi_{t}\left(z^{*}\right)\right\rangle\left\langle\psi_{t}\left(z^{*}\right)\right|\right]$.

Suppose that the system is initially at $\left|\psi_{0}\right\rangle=|\mu\rangle$, a vector in the completed set $|v\rangle$ 's. The system Hamiltonian and the coupling operator could be generally expressed by $H_{\mathrm{S}}(t)=\Sigma_{v} \omega_{v}(t)|v\rangle\langle v|$ and $L=\Sigma_{\mu \neq \nu} C_{\mu v}|\mu\rangle\langle v|$ respectively,

$$
h_{\alpha \beta}=z_{t}^{*} C_{\beta \alpha} \delta_{\beta \mu}\left\langle\alpha \mid \psi_{t}\right\rangle-\sum_{\mu^{\prime} v^{\prime}} C_{\alpha \beta *} \delta_{\alpha \mu} \delta_{\beta v^{\prime}}\left\langle v^{\prime}|\bar{O}| \mu^{\prime}\right\rangle\left\langle\mu^{\prime} \mid \psi_{t}\right\rangle
$$

and $N_{\alpha \beta} \equiv e^{-i \int_{0}^{t}\left[\omega_{\alpha}(s)-\omega_{\beta}(s)\right] d s}$. The white noise is embedded in the differences $\omega_{\alpha}-$ $\omega_{\beta} . \mathcal{F}=\sqrt{\left\langle\psi_{0}\left|M\left[\left|\psi_{t}\right\rangle\left\langle\psi_{t}\right|\right]\right| \psi_{0}\right\rangle}$ is obtained by an ensemble average over the integral results in Eq. (4). For the two-level system initially at $|1\rangle$, we can specifically write Eq. (4) as,

$$
\left\langle\psi_{0} \mid \psi_{\infty}\right\rangle-1=-\int_{0}^{\infty} Q(t) N^{*}\left\langle\psi_{0} \mid \psi_{t}\right\rangle d t
$$

where $N(t)=e^{-i \int_{0}^{t}[\omega+c(s)] d s}$.

The paper employs the biased Poissonian white noise ${ }^{18,26}, c(t)=\Sigma_{j} x_{j} \delta\left(t-t_{j}\right)$ satisfying the following statistical properties

$$
\begin{aligned}
& M[c(t)]=J W, J=M\left[x_{j}\right], \\
& M[c(t) c(s)]-M[c(t)] M[c(s)]=W M\left[x_{j}^{2}\right] \delta(t-s),
\end{aligned}
$$

where $x_{j}$ 's are noise heights. Details of numerical realization of the noise can be found in Ref. 26.

1. Breuer, H. P. \& Petruccione, F. The Theory of Open Quantum Systems. (Oxford University Press Oxford, 2002).

2. Caldeira, A. O. \& Leggett, A. J. In influence of dissipation on quantum tunneling in macroscopic systems. Phys. Rev. Lett. 46, 211-214 (1981).

3. Preskill, J. Lecture notes for physics 229: Quantum information and computation (Technical report, California Institute of Technology, 1998).

4. Gardiner, C. W. \& Zoller, P. Quantum Noise: A Handbook of Markovian and Non-Markovian Quantum Stochastic Methods with Applications to Quantum Optics. (Springer Berlin Heidelberg New York, 2004).

5. Zurek, W. H. Decoherence, einselection, and the quantum origins of the classical. Rev. Mod. Phys. 75, 715-718 (2003).

6. Vilar, J. M. G. \& Rubí, J. M. Noise Suppression by Noise. Phys. Rev. Lett. 86, 950-953 (2001).

7. Walton, D. \& Brian Visscher, K. Noise suppression and spectral decomposition for state-dependent noise in the presence of a stationary fluctuating input. Phys. Rev. E 69, 051110-051117 (2004).

8. Hahn, E. L. Spin Echoes. Phys. Rev. 80, 580-594 (1950).

9. Wiseman, H. M. Quantum theory of continuous feedback. Phys. Rev. A 49, 2133-2150 (1994).
10. Kofman, A. G. \& Kurizki, G. Unified Theory of Dynamically Suppressed Qubit Decoherence in Thermal Baths. Phys. Rev. Lett. 93, 130406-130409 (2004).

11. Viola, L., Knill, E. \& Lloyd, S. Dynamical Decoupling of Open Quantum Systems. Phys. Rev. Lett. 82, 2417-2420 (1999).

12. Santos, L. F. \& Viola, L. Enhanced Convergence and Robust Performance of Randomized Dynamical Decoupling. Phys. Rev. Lett. 97, 150501-150104 (2006).

13. Uhrig, G. S. Keeping a Quantum Bit Alive by Optimized $\pi$-Pulse Sequences. Phys. Rev. Lett. 98, 100504-100507 (2007); Exact results on dynamical decoupling by $\pi$ pulses in quantum information processes. New J. Phys. 10, 083024-083045 (2008).

14. Wu, L.-A., Kurizki, G. \& Brumer, P. Master Equation and Control of an Open Quantum System with Leakage. Phys. Rev. Lett. 102, 080405-080508 (2009).

15. Khodjasteh, K. \& Lidar, D. A. Fault-Tolerant Quantum Dynamical Decoupling. Phys. Rev. Lett. 95, 180501-180504 (2005).

16. Zhang, J., Liu, Y.-X., Zhang, W.-M., Wu, L.-A., Wu, R.-B. \& Tarn, T. J. Deterministic chaos can act as a decoherence suppressor. Phys. Rev. B 84, 214304-214312 (2011).

17. Jing, J., Wu, L.-A., You, J. Q. \& Yu, T. Feshbach projection-operator partitioning for quantum open systems: Stochastic approach. Phys. Rev. A 85, 032123-032127 (2012).

18. Spiechowicz, J., Luczka, J. \& Hanggi, P. Absolute negative mobility induced by white Poissonian noise. J. Stat. Mech.: Theor. Exp. P02044 (2013).

19. Diósi, L. \& Strunz, W. T. The non-Markovian stochastic Schrödinger equation for open systems. Phys. Lett. A 235, 569-573 (1997).

20. Diósi, L., Gisin, N. \& Strunz, W. T. Non-Markovian quantum state diffusion. Phys. Rev. A 58, 1699-1712 (1998).

21. Xiang, Z. L., Ashhab, S., You, J. Q. \& Nori, F. Hybrid quantum circuits: Superconducting circuits interacting with other quantum systems. Rev. Mod. Phys. 85, 623C653 (2013).

22. Benzi, R., Sutera, A. \& Vulpiani, A. The mechanism of stochastic resonance. J. Phys. A 14, L453 (1981).

23. Jung, P. Periodically driven stochastic systems. Phy. Rep. 234, 175-295 (1993).

24. Grifoni, M., Grifoni, M. \& Hanggi, P. Driven quantum tunneling. Phy. Rep. 304, 229-354 (1998).

25. Jing, J. \& Yu, T. Non-Markovian Relaxation of a Three-Level System: Quantum Trajectory Approach. Phys. Rev. Lett. 105, 240403-240406 (2010).

26. Kim, C., Lee, E. K., Hanggi, P. \& Talkner, P. Numerical method for solving stochastic differential equations with Poissonian white shot noise. Phys. Rev. E 76, 011109-011118 (2007).

\section{Acknowledgements}

We acknowledge grant support from the National Natural Science Foundation of China under Grant No. 11175110, the Basque Government (grant IT472-10), the Spanish MICINN (Project No. No. FIS2012-36673-C03- 03) and the Basque Country University UFI (Project No. 11/55-01-2013).

\section{Author contributions}

J.J. contributed to numerical and physical analysis and prepared the first version of the manuscript and L.-A.W. to the conception and design of this work. Both authors wrote the manuscript.

\section{Additional information}

Competing financial interests: The authors declare no competing financial interests.

How to cite this article: Jing, J. \& Wu, L. Control of decoherence with no control. Sci. Rep. 3 , 2746; DOI:10.1038/srep02746 (2013).

\footnotetext{
(c) (i) () $\odot$ This work is licensed under a Creative Commons AttributionNonCommercial-NoDerivs 3.0 Unported license. To view a copy of this license, visit http://creativecommons.org/licenses/by-nc-nd/3.0
} 